\title{
A density functional theory-based chemical potential equalisation approach to molecular polarizability
}

\author{
AMITA WADEHRA ${ }^{1}$ and SWAPAN K GHOSH* \\ Theoretical Chemistry Section, Chemistry Group, Bhabha Atomic Research Centre, Mumbai 400085 , \\ India \\ ${ }^{1}$ Present address: Department of Physics, Ohio State University, Columbus, OH 43210, USA \\ e-mail: skghosh@magnum.barc.ernet.in
}

\begin{abstract}
The electron density changes in molecular systems in the presence of external electric fields are modeled for simplicity in terms of the induced charges and dipole moments at the individual atomic sites. A chemical potential equalisation scheme is proposed for the calculation of these quantities and hence the dipole polarizability within the framework of density functional theory based linear response theory. The resulting polarizability is expressed in terms of the contributions from individual atoms in the molecule. A few illustrative numerical calculations are shown to predict the molecular polarizabilities in good agreement with available results. The usefulness of the approach to the calculation of intermolecular interaction needed for computer simulation is highlighted.
\end{abstract}

Keywords. Chemical potential equalisation; molecular polarisability; density functional theory.

\section{Introduction}

The knowledge about the response of an atomic or molecular system to an external electric field is important for an understanding of their interaction with radiation, intermolecular interaction and various other physicochemical phenomena of interest. The linear and nonlinear responses are characterised respectively by the polarizability and hyperpolarizability quantities both of which can be calculated through the evaluation of expectation values of single particle operators and hence density functional theory (DFT) ${ }^{1,2}$ which employs the single particle electron density ${ }^{3}$ as the basic variable is most suitable for this purpose from the point of view of conceptual simplicity as well as computational economy. DFT has also provided foundation $^{2,4}$ to a number of chemical concepts used widely for the understanding of chemical binding, reactivity and interaction. Thus, the chemical potential of the electron cloud has been identified ${ }^{5}$ as the electronegativity ${ }^{6}$ parameter of chemistry, the charge capacity has been linked ${ }^{7}$ with the concepts of chemical hardness ${ }^{8,9}$ and softness and the frontier orbital theory ${ }^{10}$ has been rationalised ${ }^{11}$ in terms of the density derivatives within the framework of DFT. The calculation of polarizability of many electron

*For correspondence systems using DFT has followed mainly two routes, the first one dealing ${ }^{12}$ directly with the density perturbation in an external field using variational or numerical method, while the second one makes use of a hybrid prescription ${ }^{13-15}$ exploiting the interconnection of the response functions ${ }^{16}$ with some of the conceptual developments as mentioned above.

The major features of the electron density changes during intermolecular interaction, molecule formation or interaction with external fields can, however, be often captured in terms of much simplified representation of the properties of atoms in molecules rather than using the full density function in 3-D space of the molecule. Recent years have seen an upsurge of interest in the development and extension of the conceptual framework in this direction. Thus, a perturbation theory of chemical binding has been developed ${ }^{17-20}$ where the concept of chemical potential equalisation $^{4,5}$ (CPE) has been generalised to include the concept of bond chemical potential, ${ }^{17,18}$ spinpolarised electronegativity ${ }^{19,20}$ etc., thereby incorporating the effect of charge delocalisation and spin pairing during molecule formation particularly for covalent binding. There have also been developments of other descriptions ${ }^{21}$ where the atomic dipoles arising due to distortion of the charge distribution from spherical symmetry due to bonding have been taken into account. Most widely used application of CPE pro- 
cedure however is to the calculation of partial atomic charges ${ }^{22-24}$ in molecules with the ambitious goal of development of dynamic charge models ${ }^{25-28}$ for predicting the intermolecular forces for computer simulation studies.

The purpose of the present work is to study the applicability of simple chemical potential equalisation approaches within a DFT framework to the study of response properties of molecules in external fields. For simplicity, we have restricted ourselves to the study of electric dipole polarizability only although extension to multipolar polarizability is straightforward.

There have been many attempts ${ }^{29}$ in the past to obtain molecular polarizability by adding together the contributions from the constituent atoms. The effect of the bonded environment of an atom had been accounted for in some of these additive models in an average manner by invoking the concept of atomic hybridisation dependent parameters. Attempt has also been made ${ }^{30}$ to obtain the molecular polarizability in terms of the effective atomic polarizabilities dependent on the net atomic charges on molecule formation. In an elegant approach, Applequist ${ }^{31}$ has proposed an electrostatic interaction model where the atomic response that leads to an effective atomic polarizability is not only due to the externally applied electric field but also due to an effective field arising from the induced dipole moments of all the atoms in the molecule. A major drawback of the electrostatic point dipole model has been corrected by Thole ${ }^{32}$ by modifying the dipole tensor through the use of a shape function at shorter distances. The interacting dipole model of Applequist ${ }^{31}$ however treated the molecule as a collection of polarizable points and only the induced interacting atomic dipoles contributed to the induced dipole moment and hence the polarizability. The model was subsequently extended to incorporate the interatomic charge transfer effects by Olson and Sundberg ${ }^{33}$ and was further generalised by Applequist ${ }^{34}$ himself in a series of papers as well as by others. ${ }^{35,36}$

The present work is close in spirit to these electrostatic models ${ }^{31-34}$ developed by several authors during the last two decades where the concept of effective atomic polarizabilities has been invoked to reproduce the molecular polarizability. The major new features of the present approach involve the consideration of the density changes in terms of its first two moments, viz. the atomic charge and dipole within the framework of a chemical potential equalisation procedure in contrast to a purely electrostatic model. It basically provides a simple linear response theoretic approach within DFT for the study of redistribution of the electron density due to an external field and is in the spirit of a semiempirical model for polarisation and charge transfer. Although the final equations possess classical-like appearance, the underlying physics is based on a rigorous quantum mechanical framework of DFT.

Chemical potential equalisation schemes have been attempted ${ }^{37-39}$ recently for the induced atomic charges in a molecule due to an applied field and thereby calculation of the polarizability. However, the main limitation of these works is that they are based on the charges alone and hence the polarizability in the perpendicular direction in a planar molecule is predicted to be zero. Although attempts have been made ${ }^{37}$ to use the Drude oscillator model to mimic this, a more satisfactory approach seems to lie in the consideration of the atom centered dipoles used earlier in electrostatic models. This will also unify the proposed chemical potential equalisation approach with the well developed interaction model of Applequist ${ }^{31-34}$ resulting into an integrated approach. A close connection with the distributed polarizability approaches ${ }^{40-42}$ will also be apparent.

In what follows, we first discuss the theoretical framework for density functional investigation of chemical potential equalisation of atomic constituents in molecular systems in $\S 2$ and the choice of various atomic and other (interatomic) parameters appearing in this theory is considered in $\S 3$. The linear response version of the equations corresponding to the chemical potential equalisation for molecular polarizability is discussed in $\S 4$. The results of numerical calculations for various molecular systems have been presented in $\$ 5$ which is followed by a few concluding remarks in $\S 6$.

\section{Density functional theory and effective chemical potential equalisation of atoms in molecules}

In DFT, the ground state electronic energy of a manyelectron system characterised by the external potential $\mathrm{V}(\mathbf{r})$ arising due to the nuclei and/or any other external source of electric field, can be expressed as a unique functional $E_{\mathrm{v}}[\rho(\mathbf{r})]$ of its electron density $\rho(\mathbf{r})$ and is given by,

$$
E_{\mathrm{v}}[\rho(\mathbf{r})]=\int \mathrm{d} \mathbf{r} \rho(\mathbf{r}) \mathrm{V}(\mathbf{r})+F[\rho(\mathbf{r})],
$$


where $F[\rho(\mathbf{r})]$ is a universal functional of the density consisting of kinetic, Coulomb and exchange-correlation energy components. The equilibrium density $\rho_{0}(\mathbf{r})$ is the solution of the Euler equation corresponding to the energy minimisation, viz.

$$
\mu=\frac{\delta E_{\mathrm{v}}[\rho(\mathbf{r})]}{\delta \rho(\mathbf{r})}=\mathrm{v}(\mathbf{r})+\frac{\delta F[\rho(\mathbf{r})]}{\delta \rho(\mathbf{r})},
$$

where $\mu$ is the Lagrange multiplier associated with the density normalisation $\left[\int \mathrm{d} \mathbf{r} \rho(\mathbf{r})=N_{e l}\right]$ and signifies the chemical potential of the electron cloud and hence the electronegativity $\chi$ (with a negative sign) as demonstrated by Parr et $a l^{5}$, viz.

$$
\chi=-\left(\frac{\partial E}{\partial N_{e l}}\right)_{v}=-\int \mathrm{d} \mathbf{r}\left(\frac{\delta E_{\mathrm{v}}[\rho(\mathbf{r})]}{\delta \rho(\mathbf{r})}\right)\left(\frac{\partial \rho(\mathbf{r})}{\partial N_{e l}}\right)_{v}=-\mu
$$

with $N_{e l}$ denoting the number of electrons in the system. The second derivative has also been defined ${ }^{7}$ to represent the hardness parameter $\eta$ as

$$
\eta=\frac{1}{2}\left(\frac{\partial^{2} E}{\partial N_{e l}^{2}}\right)
$$

which has further been expressed ${ }^{43}$ in terms of the density as

$$
\begin{aligned}
\eta & =\int \mathrm{d} \mathbf{r} f(\mathbf{r}) \eta(\mathbf{r}) \\
& =\int \mathrm{d} \mathbf{r} f(\mathbf{r}) \int \mathrm{d} \mathbf{r}^{\prime} \rho\left(\mathbf{r}^{\prime}\right) \eta\left(\mathbf{r}, \mathbf{r}^{\prime}\right)
\end{aligned}
$$

where $\eta(\mathbf{r})$ denotes the local hardness ${ }^{43}$ and the energy functional derivative $\eta\left(\mathbf{r}, \mathbf{r}^{\prime}\right)$ given by

$$
\eta\left(\mathbf{r}, \mathbf{r}^{\prime}\right)=\left(\frac{\delta^{2} F[\rho(\mathbf{r})]}{\delta \rho(\mathbf{r}) \delta \rho\left(\mathbf{r}^{\prime}\right)}\right)
$$

represents the hardness kernel. ${ }^{16}$ The function $f(\mathbf{r})$ used here is the Fukui function ${ }^{11}$ which has been known to play an important role in the theory of chemical reactivity and is defined as $f(\mathbf{r})=(\partial \rho(\mathbf{r}) /$ $\left.\partial N_{e l}\right)_{\vee(r)}$ although one can also use $\left(\rho(\mathbf{r}) / N_{e l}\right)$ or any other function integrating to unity in place of this function for evaluating the hardness ${ }^{44}$ through (5).

If the system is perturbed by a perturbing potential $\delta \mathrm{V}(\mathbf{r})$ so that the new potential is $\mathrm{V}(\mathbf{r})+\delta \mathrm{V}(\mathbf{r})$ and the corresponding density change is $\delta \rho(\mathbf{r})$, the energy change can be expressed ${ }^{27}$ in terms of the various functional derivatives using (1) and retaining terms up to second order as

$$
\begin{aligned}
& \Delta E= E[\rho(\mathbf{r})+\delta \rho(\mathbf{r}), \mathrm{v}(\mathbf{r})+\delta \mathrm{v}(\mathbf{r})]-E[\rho(\mathbf{r}), \mathrm{v}(\mathbf{r})] \\
&=\int \mathrm{d} \mathbf{r}\left(\frac{\delta E[\rho(\mathbf{r})]}{\delta \rho(\mathbf{r})}\right)_{V(\mathbf{r})} \delta \rho(\mathbf{r})+\int \mathrm{d} \mathbf{r}\left(\frac{\delta E[\rho(\mathbf{r})]}{\delta \mathrm{v}(r)}\right)_{\rho(\mathbf{r})} \delta \mathrm{v}(\mathbf{r}) \\
&+\frac{1}{2} \iint \mathrm{d} \mathbf{r} \mathrm{d} \mathbf{r}^{\prime}\left(\frac{\delta^{2} E[\rho(\mathbf{r})]}{\delta \rho(\mathbf{r}) \delta \rho\left(\mathbf{r}^{\prime}\right)}\right)_{V(\mathbf{r})} \delta \rho(\mathbf{r}) \delta \rho\left(\mathbf{r}^{\prime}\right) \\
&+\frac{1}{2} \iint \mathrm{d} \mathbf{r} \mathbf{d} \mathbf{r}^{\prime}\left(\frac{\delta^{2} E[\rho(\mathbf{r})]}{\delta \mathrm{v}(\mathbf{r}) \delta \mathrm{v}\left(\mathbf{r}^{\prime}\right)}\right)_{\rho(\mathbf{r})} \delta \mathrm{v}(\mathbf{r}) \delta \mathrm{v}\left(\mathbf{r}^{\prime}\right) \\
&+\iint \mathrm{d} \mathbf{r d} \mathbf{r}^{\prime}\left(\frac{\delta^{2} E[\rho(\mathbf{r})]}{\delta \rho(\mathbf{r}) \delta \mathrm{v}\left(\mathbf{r}^{\prime}\right)}\right) \delta \rho(\mathbf{r}) \delta \mathrm{v}\left(\mathbf{r}^{\prime}\right) .
\end{aligned}
$$

Analogously the change in chemical potential $\mu(=$ $\left.\mu_{0}+\Delta \mu\right)$ is given up to first order change in $\delta \rho(\mathbf{r})$ by,

$$
\begin{aligned}
\Delta \mu & =\mu-\mu_{0} \\
& =\delta \mathrm{v}(\mathbf{r})+\int \mathrm{d} \mathbf{r}^{\prime} \eta\left(\mathbf{r}, \mathbf{r}^{\prime}\right) \delta \rho\left(\mathbf{r}^{\prime}\right),
\end{aligned}
$$

as obtained by using (2) and (6) as well as the rela$\operatorname{tion}^{45}\left(\delta^{2} E / \delta \rho(\mathbf{r}) \delta \mathrm{v}\left(\mathbf{r}^{\prime}\right)\right)=\delta\left(\mathbf{r}-\mathbf{r}^{\prime}\right)$. The density change $\delta \rho(\mathbf{r})$ can also be written as a sum of contributions due to the change in the number of electrons $\mathrm{d} N_{e l}$ and the potential, viz.

$$
\delta \rho(\mathbf{r})=f(\mathbf{r}) \mathrm{d} N_{e l}+\int \mathrm{d} \mathbf{r}^{\prime} \chi_{0}\left(\mathbf{r}, \mathbf{r}^{\prime}\right) \delta \mathrm{v}\left(\mathbf{r}^{\prime}\right),
$$

involving the fukui function $f(\mathbf{r})$ and the response function $\chi_{0}\left(\mathbf{r}, \mathbf{r}^{\prime}\right)$ given by $\chi_{0}\left(\mathbf{r}, \mathbf{r}^{\prime}\right)=(\delta \rho(\mathbf{r}) / \delta \mathrm{v}$ $\left.\left(\mathbf{r}^{\prime}\right)\right)_{\mathrm{Nel}}$.

While these equations are general and applicable to any many-electron system, we here specialise to a molecular species consisting of $N$ atoms with their nuclei located at the fixed positions $\left\{R_{\alpha}\right\}$ corresponding to the equilibrium geometry of the molecule. Let the density change $\delta \rho(\mathbf{r})$ on molecule formation and due to the applied electric field $\mathbf{E}$ be partitioned in some way and be expressed as a sum of the atomic components, viz.

$$
\delta \rho(\mathbf{r})=\sum_{\alpha} \delta \rho_{\alpha}(\mathbf{r})=\sum_{\alpha} \delta \rho_{\alpha}\left(\mathbf{r}_{\alpha}\right),
$$

where $\mathbf{r}_{\alpha}=\mathbf{r}-\mathbf{R}_{\alpha}$ denotes the atom-centered coordinate with the origin located at the $\alpha$-th atom. In 
this partitioning of a molecular system into atomic subsystems, the regions may be overlapping but one can assume without any loss of generality that the density components $\delta \rho_{\alpha}(\mathbf{r})$ vanish outside the $\alpha$-th atomic region.

We now consider the $\alpha$-th atom and the region $\mathbf{r}_{\alpha}$ surrounding this atomic site $\mathbf{R}_{\alpha}$ for which the chemical potential $\mu \equiv \mu_{\alpha}\left(\mathbf{r}_{\alpha}\right)$ as given by (8) can be written as

$$
\begin{aligned}
\mu_{\alpha} & =\mu_{\alpha}^{0}+\delta \mathrm{v}\left(\mathbf{r}_{\alpha}\right)+\int d \mathbf{r}^{\prime} \eta\left(\mathbf{r}_{\alpha}, \mathbf{r}^{\prime}\right) \delta \rho\left(\mathbf{r}^{\prime}\right) \\
& =\mu_{\alpha}^{0}+\delta \mathrm{v}\left(\mathbf{r}_{\alpha}\right)+\sum_{\beta} \int \mathrm{d} \mathbf{r}_{\beta}^{\prime} \eta\left(\mathbf{r}_{\alpha}, \mathbf{r}_{\beta}^{\prime}\right) \delta \rho_{\beta}\left(\mathbf{r}_{\beta}^{\prime}\right),
\end{aligned}
$$

with the symbol $\mu_{\alpha}^{0}\left(\equiv \mu_{\alpha}(0)\right)$ denoting the chemical potential of the $\alpha$-th atom. In writing (11), the assumption that $\delta \rho_{\beta}\left(\mathbf{r}^{\prime}\right)$ is nonzero only within the region of the $\beta$-th atom has been used. Consider now the Taylor series expansions of the potential and the hardness kernel as

$$
\delta \mathrm{v}\left(\mathbf{r}_{\alpha}\right)=\delta \mathrm{v}(\alpha)+\mathbf{r}_{\alpha} \cdot \nabla_{\alpha} \delta \mathrm{v}(\alpha)+\frac{1}{2} \mathbf{r}_{\alpha}^{2} \nabla_{\alpha}^{2} \delta \mathrm{v}(\alpha)+\mathrm{K}
$$

and

$$
\begin{gathered}
\eta\left(\mathbf{r}_{\alpha}, \mathbf{r}_{\beta}^{\prime}\right)=\eta(\alpha, \beta)+\mathbf{r}_{\alpha} \cdot \nabla_{\alpha} \eta(\alpha, \beta)+\mathbf{r}_{\beta}^{\prime} \cdot \nabla_{\beta} \eta(\alpha, \beta) \\
+\frac{1}{2} \mathbf{r}_{\alpha}^{2} \nabla_{\alpha}^{2} \eta(\alpha, \beta)+\frac{1}{2} \mathbf{r}_{\beta}^{\prime 2} \nabla_{\beta}^{2} \eta(\alpha, \beta) \\
+\mathbf{r}_{\alpha} \mathbf{r}_{\beta}^{\prime} \nabla_{\alpha} \nabla_{\beta} \eta(\alpha, \beta)+\ldots
\end{gathered}
$$

where the arguments $(\alpha)$ and $(\alpha, \beta)$ as in $\delta \mathrm{v}(\alpha), \eta(\alpha$, $\beta$ ) etc. indicate that the quantities are evaluated at the atomic site $\alpha\left(\mathbf{r}_{\alpha}=0\right)$ and sites $\alpha, \beta\left(\mathbf{r}_{\alpha}=\mathbf{r}_{\beta}=0\right)$ respectively. Also the notations $\nabla_{\alpha} f(\alpha)$ and $\nabla_{\alpha} f(\alpha$, $\beta$ ) indicate derivatives of $f\left(\mathbf{r}_{\alpha}\right)$ and $f\left(\mathbf{r}_{\alpha}, \mathbf{r}_{\beta}\right)$ with respect to $\mathbf{r}_{\alpha}$, evaluated at $\mathbf{r}_{\alpha}=0$ and also $\mathbf{r}_{\beta}=0$ for the latter. Substituting these expressions (12) and (13) into (11), one obtains

$$
\begin{aligned}
\mu_{\alpha}= & \mu_{\alpha}^{0}+\delta \mathrm{v}(\alpha)+\mathbf{r}_{\alpha} \cdot \nabla_{\alpha} \delta \mathrm{v}(\alpha)+\frac{1}{2} \mathbf{r}_{\alpha}^{2} \nabla_{\alpha}^{2} \delta \mathrm{v}(\alpha) \\
& +\sum_{\beta} \eta(\alpha, \beta) \int \mathrm{d} \mathbf{r}_{\beta}^{\prime} \delta \rho_{\beta}\left(\mathbf{r}_{\beta}^{\prime}\right) \\
& +\mathbf{r}_{\alpha} \cdot \sum_{\beta} \nabla_{\alpha} \eta(\alpha, \beta) \int \mathrm{d} \mathbf{r}_{\beta}^{\prime} \delta \rho_{\beta}\left(\mathbf{r}_{\beta}^{\prime}\right) \\
& +\sum_{\beta} \nabla_{\beta} \eta(\alpha, \beta) \cdot \int \mathrm{d} \mathbf{r}_{\beta}^{\prime} \mathbf{r}_{\beta}^{\prime} \delta \rho_{\beta}\left(\mathbf{r}_{\beta}^{\prime}\right) \\
& +\frac{1}{2} \mathbf{r}_{\alpha}^{2} \sum_{\beta} \nabla_{\alpha}^{2} \eta(\alpha, \beta) \int \mathrm{d} \mathbf{r}_{\beta}^{\prime} \delta \rho_{\beta}\left(\mathbf{r}_{\beta}^{\prime}\right)
\end{aligned}
$$

$$
\begin{gathered}
-\sum_{\beta}^{2} \eta(\alpha, \beta) \int \mathrm{d} \mathbf{r}_{\beta}^{\prime} \mathbf{r}_{\beta}^{\prime 2} \delta \rho_{\beta}\left(\mathbf{r}_{\beta}^{\prime}\right) \\
+\mathbf{r}_{\alpha} \cdot \sum_{\beta} \nabla_{\alpha} \nabla_{\beta} \eta(\alpha, \beta) \int d \mathbf{r}_{\beta} \mathbf{r}_{\beta} \delta \rho_{\beta}\left(\mathbf{r}_{\beta}\right),
\end{gathered}
$$

which can be rewritten, on using the definitions of the atomic charges $q_{\beta}$ and dipoles $\mathbf{P}_{\beta}$ at the atomic site $\beta$ (with $N_{\beta}^{e l}$ denoting the corresponding number of electrons) given by

$$
q_{\beta}=-\Delta N_{\beta}^{e l}=-\int \mathrm{d} \mathbf{r}_{\beta}^{\prime} \delta \rho_{\beta}\left(\mathbf{r}_{\beta}^{\prime}\right)
$$

and

$$
\mathbf{p}_{\beta}=\int \mathrm{d}{ }_{\beta}^{\prime} \mathbf{r}^{\prime} \delta \rho_{\beta}\left(\mathbf{r}_{\beta}^{\prime}\right.
$$

in the simple form

$$
\begin{aligned}
\alpha= & \mu^{0}+\delta \mathrm{v}\left(\quad-{ }_{\alpha} \cdot \mathbf{E}_{\alpha}-{ }_{-} \mathbf{r}^{2} \nabla_{\alpha} \cdot{ }_{\alpha}\right. \\
& -\sum_{\beta} \eta(\alpha, \beta)_{\beta} \quad \mathbf{r}_{\alpha} \cdot \sum_{\beta} \nabla \eta(\alpha, \beta) q_{\beta} \\
& \underline{1}^{2} \quad \nabla_{\alpha}^{2} \eta(\alpha, \beta) q \\
& +\sum_{\beta} \nabla_{\beta} \eta(\alpha, \beta) \mathbf{p}_{\beta}+\mathbf{r}_{\alpha} \cdot \sum_{\beta} \nabla_{\alpha} \nabla_{\beta} \eta(\alpha, \beta) \mathbf{p}_{\beta},
\end{aligned}
$$

where terms involving quadrupole moments have been neglected. Here $\mathbf{E}_{\alpha}\left(=-\nabla_{\alpha} \delta \mathrm{V}(\alpha)\right)$ represents the electric field at the atomic site $\alpha$. This equation essentially expresses the chemical potential of an atom in a molecular species simplified under the assumption of superposed atomic site densities and approximated in terms of its first two moments, viz. the atomic charges and the dipole moments denoted respectively by $q_{\alpha}$ and $\mathbf{p}_{\alpha}$ for the $\alpha$-th atomic site. Thus, in this model a molecule is a collection of atom centers containing point charges and dipoles, the magnitudes of which are determined through the chemical potential equalisation of DFT by equating $\mu_{\alpha}$ as embodied in the above equation for all the atoms.

Now, since this equation is valid for any value of $\mathbf{r}_{\alpha}$ and the left side is a constant, one can generate two sets of equations by equating on both sides the constant terms and the terms linear in $\mathbf{r}_{\alpha}$ respectively, viz.

$$
\mu_{\alpha}=\mu_{\alpha}^{0}+\delta v(\alpha)-\sum_{\beta} \eta(\alpha, \beta) q_{\beta}+\sum_{\beta} \nabla_{\beta} \eta(\alpha, \beta) \mathbf{p}_{\beta}
$$


and

$$
0=\mathbf{E}_{\alpha}+\sum_{\beta} \nabla_{\alpha} \eta(\alpha, \beta) q_{\beta}-\sum_{\beta} \nabla_{\alpha} \nabla_{\beta} \eta(\alpha, \beta) \mathbf{p}_{\beta}
$$

which can be rewritten by separating out the $\beta=\alpha$ terms as

$$
\begin{aligned}
\mu_{\alpha}=\mu_{\alpha}^{0} & +\delta v(\alpha)-\eta(\alpha, \alpha) q_{\alpha} \\
& -\sum_{\beta \neq \alpha} \eta(\alpha, \beta) q_{\beta}+\sum_{\beta \neq \alpha}\left[\nabla_{\beta} \eta(\alpha, \beta)\right] \mathbf{p}_{\beta},
\end{aligned}
$$

and

$$
\begin{aligned}
0= & \mathbf{E}_{\alpha}+\sum_{\beta \neq \alpha}\left[\nabla_{\alpha} \eta(\alpha, \beta)\right] q_{\beta}-\left[\left.\nabla_{\alpha} \nabla_{\beta} \eta(\alpha, \beta)\right|_{\alpha=\beta}\right] \mathbf{p}_{\alpha} \\
& -\sum_{\beta \neq \alpha}\left[\nabla_{\alpha} \nabla_{\beta} \eta(\alpha, \beta)\right] \mathbf{p}_{\beta},
\end{aligned}
$$

for $\alpha=1, \ldots, N$. Here the diagonal $(\alpha=\beta)$ contributions from the quantities $\nabla_{\beta} \eta(\alpha, \beta)$ and $\nabla_{\alpha} \eta(\alpha, \beta)$ vanish as the charges and dipoles at the same sites do not interact with each other in this point charge dipole approximation and hence the potential and the field generated at site $\alpha$ due to the dipole $\mathbf{p}_{\alpha}$ and the charge $q_{\alpha}$ respectively are omitted.

Since at equilibrium the chemical potentials of all the atoms are equalised, one can obtain a set of $N-1$ equations by equating $\mu_{\alpha}=\mu_{\beta}$ given by (20) for all the atoms. The $N$-th equation is provided by the charge conservation (neutrality for a neutral molecule) $\sum q_{\alpha}=0$. This set of $N$ scalar equations together with $N$ vector equations corresponding to (21) determine the $N$ scalar atomic charges $\left\{q_{\alpha}\right\}$ and $N$ vector atomic dipoles $\left\{\mathbf{p}_{\alpha}\right\}$.

\section{Choice of the atomic and interatomic parameters}

While the parameters appearing in (20) and (21) can be obtained in principle from a suitable form of the hardness kernel $\eta\left(\mathbf{r}, \mathbf{r}^{\prime}\right)$ used in (13) for approximation, it is often more appealing to identify them from physical considerations by comparing the equations with those of the so called atoms-in-molecules approach or the atom-charge-dipole interaction model for binding and polarizability respectively. Thus, one can identify the parameters as follows. The scalar hardness kernel $\eta(\alpha, \beta)$ used here resembles the atomin-molecule hardness matrix $\left\{\eta_{\alpha \beta}\right\}$ introduced and widely investigated by Nalewajski ${ }^{46}$ and can be modeled along similar lines. ${ }^{47}$ The other vector and tensor quantities introduced here, however, have no such precedence and are modeled through electrostatic analogy. For this purpose, we have used a slightly different and simplified notation for the hardness kernels, viz. the scalar $\eta(\alpha, \beta)=\eta_{\alpha \beta}^{0,0}$, the vectors $\nabla_{\beta} \eta(\alpha, \beta)=\vec{\eta}_{\alpha \beta}^{0,1}, \quad \nabla_{\alpha} \eta(\alpha, \beta)=\vec{\eta}_{\alpha \beta}^{1,0}$ and the tensor $\nabla_{\alpha} \nabla_{\beta} \eta(\alpha, \beta)=\overleftrightarrow{\eta}_{\alpha \beta}^{1,1}$, where the first or second superscripts as unity denotes the derivative with respect to the first or second argument respectively, while the superscript zero indicates no derivative.

Thus, the diagonal element $\eta(\alpha, \alpha)\left(=\eta_{\alpha \alpha}^{0,0}\right)$ of the scalar kernel $\eta(\alpha, \beta)$ is clearly the hardness $\eta_{\alpha}^{0}$ of the $\alpha$-th atomic species while the off-diagonal element $\eta_{\alpha \beta \beta}^{0,0}$ corresponds to the charge-charge interaction and hence can be represented by the Coulomb potential between the sites $\alpha$ and $\beta$ in terms of their internuclear distance $R_{\alpha \beta}=\left|\mathbf{R}_{\alpha \beta}\right|$ with $\mathbf{R}_{\alpha \beta}=\mathbf{R}_{\alpha}-\mathbf{R}_{\beta}$ or in a better way by the Mataga-Nishimoto-Ohno formula $a^{47,48}$ of semiempirical quantum chemistry. Thus, one has

$$
\begin{aligned}
& \eta(\alpha, \alpha)=\eta_{\alpha \alpha}^{0,0}=\eta_{\alpha}^{0} \\
& \eta(\alpha, \beta)=\eta_{\alpha \beta}^{0,0}=1 / \varepsilon R_{\alpha \beta} \\
& \quad \text { Coulomb approximation }) \\
& \eta(\alpha, \beta)=\eta_{\alpha \beta}^{0,0}=1 /\left(R_{\alpha \beta}+a_{\alpha \beta}\right) ; a_{\alpha \beta}=2 /\left(\eta_{\alpha}^{0}+\eta_{\beta}^{0}\right)
\end{aligned}
$$

$$
\text { (Mataga-Nishimoto-Ohno formula) }
$$

where $\varepsilon$ represents a measure of the dielectric constant of the electron cloud medium for the Coulomb interaction approximation. One can also rewrite (24) in the form of (23) by defining an effective dielectric constant $\varepsilon_{\alpha \beta}$ specific to the bonded atom pair $\alpha$ and $\beta$ as

$$
\eta_{\alpha \beta}^{0,0}=1 / \varepsilon_{\alpha \beta} R_{\alpha \beta} ; \quad \varepsilon_{\alpha \beta}=\left(1+a_{\alpha \beta} / R_{\alpha \beta}\right) .
$$

The vector quantities $\nabla_{\beta} \eta(\alpha, \beta)=\vec{\eta}_{\alpha \beta}^{0,1}$ and $\nabla_{\alpha} \eta(\alpha$, $\beta)=\vec{\eta}_{\alpha \beta}^{1,0}$ correspond to charge-dipole interaction representing essentially the potential at the $\alpha$-th atom due to a unit point dipole at the $\beta$-th atom and the field at the $\alpha$-th atom due to a unit point charge at the $\beta$-th atom respectively. One can thus write

$$
\nabla_{\beta} \eta(\alpha, \beta)=\vec{\eta}_{\alpha \beta}^{0,1}=\mathbf{R}_{\alpha \beta} / \varepsilon R_{\alpha \beta}^{3}
$$

and

$$
\nabla_{\alpha} \eta(\alpha, \beta)=\vec{\eta}_{\alpha \beta}^{1,0}=-\mathbf{R}_{\alpha \beta} / \varepsilon R_{\alpha \beta}^{3}
$$


for $\alpha \neq \beta$ and zero otherwise as already indicated. Clearly, one has the result $\nabla_{\beta} \eta(\alpha, \beta)=-\nabla_{\alpha} \eta(\alpha, \beta)$, i.e., $\vec{\eta}_{\alpha \beta}^{0,1}=-\vec{\eta}_{\alpha \beta}^{1,0}$ and $\vec{\eta}_{\alpha \alpha}^{0,1}=\vec{\eta}_{\alpha \alpha}^{1,0}=0$.

Analogously, the tensor quantity $\nabla_{\alpha} \nabla_{\beta} \eta(\alpha, \beta)$ corresponds to the dipole-dipole interaction representing essentially the field at the $\alpha$-th atom due to a unit point dipole at the $\beta$-th atom and hence can be expressed by the dipole-dipole tensor of electrostatics as

$$
\nabla_{\alpha} \nabla_{\beta} \eta(\alpha, \beta)=\eta_{\alpha \beta}^{\mathrm{t}_{1,1}}=-\frac{1}{\varepsilon R_{\alpha \beta}^{3}}\left[1-\frac{3}{R_{\alpha \beta}^{2}} \mathbf{R}_{\alpha \beta} \mathbf{R}_{\alpha \beta}\right] .
$$

The corresponding diagonal term $\left.\nabla_{\alpha} \nabla_{\beta} \eta(\alpha, \beta)\right|_{\alpha=\beta}$ can be easily interpreted as the inverse polarizability as is evident by considering (19) or (21) for a single atom, where the off-diagonal terms are absent and hence one has $0=\mathbf{E}_{\alpha}-\left.\nabla_{\alpha} \nabla_{\beta} \eta(\alpha, \beta)\right|_{\alpha=\beta} \mathbf{p}_{\alpha}$. One can thus write,

$$
\left.\nabla_{\alpha} \nabla_{\beta} \eta(\alpha, \beta)\right|_{\alpha=\beta}=\overleftrightarrow{\eta}_{\alpha \alpha}^{1,1}=\overleftrightarrow{\alpha}_{\alpha}^{-1}
$$

where $\overleftrightarrow{\alpha}_{\alpha}$ represents the polarizability tensor of the $\alpha$-th atom. This quantity can be interpreted as the hardness parameter for the induction of atomic dipole just as $\eta$ is the hardness for charge transfer.

It may be noted that here the parameters $\eta_{\alpha \beta}^{0,0}$, $\vec{\eta}_{\alpha \beta}^{0,1}=-\vec{\eta}_{\alpha \beta}^{1,0}$ and $\overleftrightarrow{\eta}_{\alpha \beta}^{1,1}$ denoting respectively the scalar, vector and tensor quantities, with the superscripts 0 and 1 referring to charge and dipole respectively, are given by the simple expressions of electrostatics as indicated above. As has already been pointed out, the expression given by (25) for $\eta_{\alpha \beta}^{0,0}$ in the case of bonded atoms is also of the same form but with an effective dielectric constant specific to an atom pair.

\section{Chemical potential equalisation, linear response and molecular polarizability}

It is well known that a molecule formation is associated with equalisation of chemical potential of the constituent atoms mediated through electron density redistribution. When an external electric field is applied to the molecule, a further reorganisation of the electron density takes place and modified charges and dipoles are induced at each site. The chemical potential equalisation equations in the presence of the field provides equations for evaluation of these quantities. For this purpose, we first rewrite (20) representing the effective chemical potential (of the $\alpha$-th atom) in the already discussed notation as

$$
\begin{gathered}
\mu_{\alpha}=\mu_{\alpha}^{0}-\eta_{\alpha}^{0} q_{\alpha}+\left(\phi_{\alpha}^{0}-\sum_{\beta=1}^{N} \eta_{\alpha \beta}^{0,0} q_{\beta}+\sum_{\beta=1}^{N} \vec{\eta}_{\alpha \beta}^{0,1} \cdot \mathbf{p}_{\beta}\right), \\
\alpha=1,2, \ldots, N
\end{gathered}
$$

where the notation $\Sigma^{\prime}$ indicates omission of $\beta=\alpha$ term in the summation, $\phi_{\alpha}^{0}=\delta \mathrm{v}(\alpha)$ is the potential at the $\alpha$-th atomic site due to an external field $\mathbf{E}^{0}$ which is considered to be uniform for the purpose of polarizability calculation here, but can be in general a position dependent field. Also $\mu_{\alpha}^{0}$ and $\eta_{\alpha}^{0}$ represent the chemical potential and hardness parameters of the $\alpha$-th atom in a suitable neutral atom reference state (prior to change transfer) for the molecule.

Equating the effective chemical potential as given by (20) or (30) for all the sites, one obtains $(N-1)$ equations which are linear in the charges $\left\{q_{\alpha}\right\}$ and the dipole moments $\left\{\mathbf{p}_{\alpha}\right\}$ of the atomic sites. Another equation is provided by the charge neutrality of the molecule, viz.

$$
\sum_{\alpha=1}^{N} q_{\alpha}=0
$$

where the right hand side which is zero here for a neutral molecule, is to be replaced by the net charge of the molecule for an ionic species. It may be noted that these atomic charges and dipoles for the molecule in presence of the field, include contributions from interatomic charge transfer during molecule formation (from the reference state) in addition to the field-induced contributions. Writing these equations for the unperturbed molecule (using $\phi_{\alpha}^{0}=0$ and $\mathbf{E}_{\alpha}=\mathbf{E}^{0}=0$, one obtains equations for the charges and dipoles due to molecule formation from the reference state. Subtraction of the corresponding equations in the two sets leads to $N$ equations involving the induced charges and dipoles $\left\{\delta q_{\alpha}\right\}$ and $\left\{\delta \mathbf{p}_{\alpha}\right\}$ respectively. The final equations can be written in matrix form as

$$
\sum_{\beta=1}^{N} b_{\alpha \beta}^{0,0} \delta q_{\beta}+\sum_{\beta=1}^{N} \vec{c}_{\alpha \beta}^{0,1} \delta \mathbf{p}_{\beta}=d_{\alpha}, \alpha=1,2, \ldots, N
$$

where the coefficients $b_{\alpha \beta}^{0,0}, \vec{c}_{\alpha \beta}^{0,1}$ and $d_{\alpha}$ are given by

$$
b_{\alpha \beta}^{0,0}=\left(\eta_{\alpha \beta}^{0,0}-\eta_{1 \beta}^{0,0}\right)+\delta_{\alpha 1},
$$




$$
\begin{aligned}
\vec{c}_{\alpha \beta}^{0,1} & =\left(\vec{\eta}_{\alpha \beta}^{0,1}-\vec{\eta}_{1 \beta}^{0,1}\right), \\
d_{\alpha} & =\left(\phi_{1}^{0}-\phi_{\alpha}^{0}\right),
\end{aligned}
$$

with $\eta_{\alpha \beta}^{0,0}=\eta_{\alpha}^{0}$ and $\vec{\eta}_{\alpha \alpha}^{0,1}=0$ as implied. The results $b_{\alpha \beta}^{0,0}=1, \vec{c}_{\alpha \beta}^{0,1}=0$ and $d_{\alpha}=0$ for $\alpha=1$ follow directly from the general results.

Analogously, (21) can be written for the induced dipoles as

$$
\begin{aligned}
\delta \mathbf{p}_{\alpha} & =\overleftrightarrow{\alpha}_{\alpha} \cdot\left(\mathbf{E}^{0}-\sum_{\beta=1}^{N} \mathfrak{\eta}_{\alpha \beta}^{1,1} \delta q_{\beta}-\sum_{\beta=1}^{N} \overleftrightarrow{\eta}_{\alpha \beta}^{1,1} \delta \mathbf{p}_{\beta}\right), \\
\alpha & =1,2, \ldots, N
\end{aligned}
$$

which can be rewritten in the general matrix form as

$$
\sum_{\beta=1}^{N} \stackrel{r}{h_{\alpha \beta}^{1,0}} \delta q_{\beta}+\sum_{\beta=1}^{N}{ }_{k \alpha \beta}^{\mathrm{t}} \cdot \delta \mathbf{p}_{\beta}=\stackrel{r}{r}, \quad \alpha,=1,2, \mathrm{~K}, N,
$$

with the coefficients $\overrightarrow{\mathrm{h}}_{\alpha \beta}^{1,0}, \vec{k}_{\alpha \beta}^{0,1}$ and $\overrightarrow{\mathrm{s}}_{\alpha}$ given by

$$
\begin{aligned}
& \mathbf{h}_{\alpha \beta}^{1,0}=\overleftrightarrow{\alpha}_{\alpha} \cdot \overleftrightarrow{\eta}_{\alpha \beta}^{1,0}, \\
& \overleftrightarrow{k}_{\alpha \beta}^{1,1}=\overleftrightarrow{\alpha}_{\alpha} \cdot \overleftrightarrow{\eta}_{\alpha \beta}^{1,1}, \\
& \vec{s}_{\alpha}=\overleftrightarrow{\alpha}_{\alpha} \cdot \mathbf{E}^{0} .
\end{aligned}
$$

where use has been made of the fact that $\vec{\eta}_{\alpha \alpha}^{1,0}=0$ and $\overleftrightarrow{\eta}_{\alpha \alpha}^{1,1}=\overleftrightarrow{\alpha}_{\alpha}^{-1}$. The results $\vec{h}_{\alpha \alpha}^{1,0}=0$ and $\overleftrightarrow{k}_{\alpha \alpha}^{1,1}=\overleftrightarrow{1}$ follow automatically for the case of $\alpha=\beta$ from the general expressions in (38) and (39) respectively.

Thus, (32) and (37) with the coefficients defined by (33)-(35) and (38)-(40), constitute a set of $4 N$ linear equations for $4 N$ unknowns (charge and three components of the dipole vector for each of the $N$ atomic sites) which can be solved to calculate the induced charges $\delta q_{\alpha}$ and dipoles $\delta \mathbf{p}_{\alpha}$ at each ( $\alpha$-th) atomic site in the molecule. The net induced dipole moment is then calculated by evaluating the expression,

$$
\delta \mathbf{p}_{m o l}=\sum_{\alpha=1}^{N}\left(\mathbf{R}_{\alpha} \delta q_{\alpha}+\delta \mathbf{p}_{\alpha}\right),
$$

and hence the molecular polarizability components can be calculated as

$$
\begin{aligned}
& \alpha_{x x}=\left(\overrightarrow{\mathbf{p}}_{m o l}\right)_{x} / E_{x}^{0} ; \quad \alpha_{y y}=\left(\delta \mathbf{p}_{\mathrm{mol}}\right)_{y} / E_{y}^{0} ; \\
& \alpha_{z z}=\left(\delta \mathbf{p}_{m o l}\right)_{z} / E_{\mathrm{z}}^{0}
\end{aligned}
$$

\section{Results and discussion}

Thus, with all the parameters defined, we now have one scalar equation (32) and one vector equation (37) corresponding to each of the $N$ atoms for determining the induced charges $\left\{\delta q_{\alpha}\right\}$ and dipoles $\left\{\delta \mathbf{p}_{\alpha}\right\}$ at these atomic sites in the molecule. The atomic parameters entering in these equations consist of the polarizability and hardness quantities of the atoms of the molecule. Although one can use available experimental or even calculated values of the atomic polarizability, when the field is applied to the molecule, it is the bonded atom which gets polarised and hence the polarizability should be effectively that of the bonded atoms which is less than that of the free atoms. We, therefore, propose to employ a multiplicative correction factor which we presently determine empirically. Analogously, the hardness parameters defined in (4) and calculated as $(I-A) / 2$, within the finite difference approximation, from the experimental ionisation potential $(I)$ and the electron affinity $(A)$ values for the free atoms are corrected to partially account for the bonded atoms with increased hardness. For simplicity, we assume this factor to be inverse of that used for the polarizability in view of the inverse proportionality observed ${ }^{49}$ between these two quantities. The factors for polarizability, however, are assumed to be unity in the perpendicular direction for a planar molecule and for the two perpendicular directions for a linear molecule.

Illustrative numerical calculations of the mean polarizability of several diatomic as well as polyatomic molecules have been carried out using the experimental values of the atomic polarizability and hardness parameters. However, as already indicated the polarizabilities have been scaled by a constant factor to account for the reduction in polarizability in bond formation with a parallel increase in hardness implemented through inverse of this scale factor, thus introducing only one scale parameter. The site-site charge-charge hardness $\eta_{\alpha \beta}^{0,0}$ given by (24) (or (25) in the form of an effective dielectric constant $\varepsilon_{\alpha \beta}$ ) which corresponds to the Mataga-Nishimoto-Ohno formula has been assumed only for the bonded atom pairs. For non-bonded pairs, this hardness quantity has been given by the Coulomb approximation (23), with an empirically assumed value of the dielectric constant. Thus essentially one employs the Coulomb approximation in both cases but the dielectric constant for nonbonded atom pair is an empirical parameter while the same is an effective dielectric constant for the bonded atom pair for bonded atoms. 
The other hardness quantities $\vec{\eta}_{\alpha \beta}^{0,1}, \quad \vec{\eta}_{\alpha \beta}^{1,0}$ and $\overleftrightarrow{\eta}_{\alpha \beta}^{1,1}$ are calculated using the expressions given by (26)-(29). These quantities determine the coefficients appearing in the linear equations (32) and (37) which are solved numerically by using the standard matrix inversion techniques to determine the induced atomic charges $\left\{\delta q_{\alpha}\right\}$ and dipole moments $\left\{\delta \mathbf{p}_{\alpha}\right\}$ as a function of the field strength. The mean polarizability is then calculated using these calculated charges and dipoles.

The polarizability and hardness parameters as well as the correction factor $\alpha_{f a c}$ for each atom as used in the calculation has been given in table 1 . These values of $\alpha_{f a c}$ are chosen so as to reproduce the experimental polarizabilities of the corresponding homonuclear diatomic molecules. Two sets of calculations have been carried out corresponding to two choices of the dielectric constant. Thus, the first corresponds to the use of an effective $\varepsilon$ as given by (25), while

Table 1. Atomic parameters (in a.u.) used in the calculation.

\begin{tabular}{lccc}
\hline Atom & Polarizability $\alpha$ & Hardness $\eta$ & $\alpha_{f a c}$ \\
\hline $\mathrm{H}$ & 4.5 & 0.235 & 0.45 \\
$\mathrm{C}$ & 11.9 & 0.184 & 0.3 \\
$\mathrm{O}$ & 5.41 & 0.223 & 0.3 \\
$\mathrm{Cl}$ & 14.7 & 0.172 & 0.5 \\
\hline
\end{tabular}

Table 2. Polarizability $\alpha$ (in a.u.) of simple molecular systems.

\begin{tabular}{|c|c|c|}
\hline Molecule & Calculated $\alpha$ & Experimental $\alpha$ \\
\hline $\mathrm{HCl}$ & $\begin{array}{l}17 \cdot 11^{\text {(a) }} \\
17 \cdot 12^{\text {(b) }}\end{array}$ & $17 \cdot 76$ \\
\hline $\mathrm{CO}$ & $\begin{array}{l}12.92 \\
13.21\end{array}$ & $13 \cdot 17$ \\
\hline $\mathrm{H}_{2} \mathrm{O}$ & $\begin{array}{l}9.72 \\
8.54\end{array}$ & 9.79 \\
\hline $\mathrm{CO}_{2}$ & $\begin{array}{l}16 \cdot 65 \\
17 \cdot 42\end{array}$ & $19 \cdot 66$ \\
\hline $\mathrm{C}_{2} \mathrm{H}_{2}$ & $\begin{array}{l}21.99 \\
22.25\end{array}$ & $22 \cdot 49$ \\
\hline $\mathrm{HCHO}$ & $\begin{array}{l}15 \cdot 02 \\
15 \cdot 20\end{array}$ & 18.92 \\
\hline $\mathrm{CH}_{4}$ & $\begin{array}{l}20 \cdot 70 \\
20 \cdot 82\end{array}$ & 17.52 \\
\hline $\mathrm{CCl}_{4}$ & $\begin{array}{l}79 \cdot 03 \\
79 \cdot 29\end{array}$ & $70 \cdot 93$ \\
\hline $\mathrm{CH}_{3} \mathrm{Cl}$ & $\begin{array}{l}34 \cdot 38 \\
34 \cdot 51\end{array}$ & $31 \cdot 2$ \\
\hline $\mathrm{CHCl}_{3}$ & $\begin{array}{l}63.56 \\
63.77\end{array}$ & $64 \cdot 17$ \\
\hline
\end{tabular}

the second one uses the same value of $\varepsilon=2.0$ for all the molecules considered here. The calculated mean polarizabilities for a number of diatomic and polyatomic molecules are shown in table 2 alongwith the experimental results and it is clear that the agreement is quite good. It is also apparent from the two sets of the results that the calculated polarizabilities are not very sensitive to the value of the dielectric constant used. At present one empirical parameter has been used for each atom but we hope to rationalise this in terms of the different properties and charges on the atoms and also possibly the anisotropy of the bonded atom.

\section{Concluding remarks}

The present work reports a DFT-based chemical potential equalisation approach for the calculation of response properties of molecules in an external field. The chemical potential equalisation is done in two stages, viz. the first one during the molecule formation from isolated atoms and the second one after the external field is applied. The induced charges and dipoles at the atomic sites are calculated from a set of linear equations derived here. A unified picture, supported by a sound theoretical framework, that has thus emerged encompasses all the earlier empirical approaches for the evaluation of molecular polarizability from the atomic ones.

The calculated polarizabilities obtained here from the chosen atomic parameters are found to be in good agreement with the available experimental values. The importance of the work lies in the ease with which one can calculate the response properties which can ultimately be used for the calculation of intermolecular interactions or forces using this method. This simplicity can be immensely time saving in molecular simulation studies. Further work in this direction is in progress.

\section{Acknowledgments}

It is a pleasure to thank Professor B M Deb for his advice, interest and encouragement. AW would like to thank the Jawaharlal Nehru Centre for Advanced Scientific Research, Bangalore, India for a Summer Research Fellowship. She also thanks the Bhabha Atomic Research Centre for providing the facilities for work during her stay at Mumbai, and to Dr J P Mittal and Dr T Mukherjee for their interest and encouragement. 


\section{References}

1. Hohenberg P and Kohn W 1964 Phys. Rev. B136 864; Kohn W and Sham L J 1965 Phys. Rev. A140 1133

2. Parr R G and Yang W 1989 Density functional theory of atoms and molecules (New York: Oxford University Press)

3. March N H and Deb B M (eds) 1987 Single particle density in physics and chemistry (New York: Academic Press)

4. Parr R G and Yang W 1995 Annu. Rev. Phys. Chem. 46701

5. Parr R G, Donnelly R A, Levy M and Palke W E 1978 J. Chem. Phys. 683801

6. Sen K D and Jorgensen C K (eds) 1987 Electronegativity: Structure and bonding, vol 66 (Berlin: Springer-Verlag)

7. Parr R G and Pearson R G 1983 J. Am. Chem. Soc. 1057512

8. Pearson R G 1973 Hard and soft acids and bases (Stroudsville, PA: Dowden, Hutchinson and Ross)

9. Sen K D (ed.) 1982 Chemical hardness, structure and bonding, vol 80 (Berlin: Springer-Verlag)

10. Fukui K 1982 Science 218747

11. Parr R G and Yang W 1984 J. Am. Chem. Soc. 106 4049

12. Ghosh S K and Deb B M 1982 Chem. Phys. 71295

13. Ghanty T K and Ghosh S K 1994 J. Am. Chem. Soc. 1168801

14. Ghanty T K and Ghosh S K 1994 J. Phys. Chem. 98 9197

15. Vela A and Gazquez J L 1990 J. Am. Chem. Soc. 112 1490

16. Berkowitz M and Parr R G 1988 J. Chem. Phys. 882554

17. Ghosh S K and Parr R G 1987 Theor. Chem. Acta 72 379

18. Ghanty T K and Ghosh S K 1991 J. Phys. Chem. 95 6512; 1992 Inorg. Chem. 311951

19. Ghosh S K 1994 Int. J. Quant. Chem. 49239

20. Ghanty T K and Ghosh S K 1994 J. Am. Chem. Soc. 1163943

21. Ghanty T K and Ghosh S K 1994 J. Phys. Chem. 98 1840

22. Mortier W, Ghosh S K and Shankar S 1986 J. Am. Chem. Soc. 1084315

23. Ghanty T K and Ghosh S K 1992 J. Mol. Struct. Theochem. 27683

24. Itskowitz P and Berkowitz M 1998 J. Phys. Chem. A101 5687; 1998 J. Phys. Chem. A102 4808

25. Rappe A K and Goddard III W A 1991 J. Phys. Chem. 953358

26. Rick S W, Stuart S J and Berne B J 1994 J. Chem. Phys. 1016141

27. York D M and Yang W 1996 J. Chem. Phys. 104159
28. Stern H A, Kaminski G A, Banks J L, Zhou R, Berne B J and Freisner R A 1999 J. Phys. Chem. B103 4730; Banks J L, Kaminski G A, Zhou R, Mainz D T, Berne B J and Freisner R A 1999 J. Chem. Phys. 110 741; Stern H A, Rittner F, Berne B J and Freisner R A 2001 J. Chem. Phys. 1152237

29. Miller K J 1990 J. Am. Chem. Soc. 112 8533, 8543; Miller K J and Savchik J A 1979 J. Am. Chem. Soc. 1017206

30. No K T, Cho K H, John M S and Scheraga H A 1993 J. Am. Chem. Soc. 1152005

31. Applequist J 1977 Acc. Chem. Res. 10 79; Applequist J, Carl J R and Fung K K 1972 J. Am. Chem. Soc. 94 2952

32. Thole B T 1981 Chem. Phys. 59 341; van Duijnen P T and Swart M 1998 J. Phys. Chem. 1022399

33. Olson M L and Sundberg K R 1978 J. Chem. Phys. 695400

34. Applequist J 1985 J. Chem. Phys. 83 809; Applequist J 1993 J. Phys. Chem. 97 6016; Shanker B and Applequist J 1996 J. Phys. Chem. A100 10834; Bode K A and Applequist J 1996 J. Phys. Chem. 100 17820; Applequist J 1998 J. Phys. Chem. A102 7723

35. Jensen L, Astrand P O, Sylvester-Hvid K O and Mikkelsen K V 2000 J. Phys. Chem. A104, 1563; Jensen L, Schmidt O H, Mikkelsen K V and Astrand P O 2000 J. Phys. Chem. B104 10462

36. Jensen L, Astrand P O and Mikkelsen K V 2001 Int. J. Quantum Chem. 84513

37. Dinur U 1990 J. Phys. Chem. 97 7894; 1994 J. Mol. Struct. Theochem. $\mathbf{3 0 3} 227$

38. Itskowitz P and Berkowitz M 1998 J. Chem. Phys. 10910142

39. Chelli R, Procacci P, Righini R and Califano S 1999 J. Chem. Phys. 1118569

40. Stone A J 1985 Mol. Phys. 561065

41. Stone A J 1996 The theory of intermolecular forces (Oxford: Clarendon)

42. in het Panhuis M, Popelier P L A, Munn R W and Angyan J G 2001 J. Chem. Phys. 1147951

43. Berkowitz M, Ghosh S K and Parr R G 1985 J. Am. Chem. Soc. 107 6811; Ghosh S K 1990 Chem. Phys. Lett. 17277

44. Harbola M K, Chattaraj P K and Parr R G 1991 Israel J. Chem. 31395

45. Gazquez J L and Vela A 1988 Int. J. Quantum Chem. S22 71

46. Nalewajski R F, Korchowiec J and Michalak A 1996 Top. Curr. Chem. 18325

47. Nalewajski R F, Korchowiec J and Zhou Z 1988 Int. J. Quantum. Chem. Symp. 22349

48. Mataga N and Nishimoto K 1957 Z. Phys. Chem. 13 140; Ohno K 1968 Theor. Chim. Acta 10111

49. Ghanty T K and Ghosh S K 1993 J. Phys. Chem. 97 4951 